\title{
Rate of cardiovascular recovery to combined or separate orthostatic and mental challenges
}

\author{
Nandu Goswami ${ }^{\text {a,* }}$, Helmut Karl Lackner ${ }^{\mathrm{b}}$, Ilona Papousek ${ }^{\mathrm{c}}$, Daniela Jezova ${ }^{\mathrm{d}}$, \\ Helmut Hinghofer-Szalkay ${ }^{\mathrm{a}, \mathrm{b}}$, Jean-Pierre Montani ${ }^{\mathrm{e}}$ \\ a Institute of Physiology, Center of Physiological Medicine, Medical University, Graz, Austria \\ b Institute of Adaptive and Spaceflight Physiology, Wormgasse 9, 8010 Graz, Austria \\ c Department of Psychology, Karl Franzens University, 8010 Graz, Austria \\ d Institute of Experimental Endocrinology, Slovak Academy of Science, Bratislava, Slovakia \\ e Department of Medicine/Physiology, University of Fribourg, Switzerland
}

Post stress neurovascular responses induced by physical and mental stress are poorly understood. We investigated the time course of cardiovascular and autonomic recovery, induced by orthostatic and mental challenge, using passive head up tilt (HUT) and mental arithmetic (MA), respectively, when applied singly (MA, HUT) or in combination (MA + HUT). Fifteen healthy males participated in three protocols: HUT, MA and combined MA + HUT, with sessions randomized and 2 weeks apart. Post stress responses were studied in the first $10 \mathrm{~min}$ (early; cardiovascular only) and $30 \mathrm{~min}$ (late), in $2.5 \mathrm{~min}$ epochs. A detailed analysis of early period was done in $30 \mathrm{~s}$ epochs. Within the first 2.5 min recovery, time courses of heart rate, stroke volume and cardiac output differed significantly, particularly when comparing HUT vs. MA and MA + HUT vs. MA. Additionally, heart rate response differed in HUT vs. MA + HUT. No differences in hemodynamic recovery were seen during the next $2.5 \mathrm{~min}$. Late responses of heart rate and cardiac output showed significantly lower values as compared to baseline, especially for HUT and MA+HUT. Recovery of hemodynamic responses, either due to single or combined stress challenges, showed stressor- and timedependent patterns. Our data provide useful information regarding why longer recovery periods must be assessed and provide novel insights regarding recovery of physical and mental stressors. This may have clinical implications in the development of cardiovascular diseases such as hypertension or myocardial ischemia.

\section{Introduction}

In recent years, physiological recovery after stressors has gained recognition as a decisive element in theories that explain the link between stress and disease, specifically cardiovascular disease (e.g. Pieper, $2005 \# 1930\}$ ). Nevertheless, as opposed to the conventional reactivity hypothesis that emphasizes responses during stressors (Lovallo and Gerin, 2003), to date only a small proportion of research has explicitly addressed the issue of recovery (Chida and Hamer, 2008; Linden et al., 1997; Papousek et al., in press; Pieper and Brosschot, 2005). Among other things, this may be due to the fact that relatively little is known about the behavior of different physiological variables after termination of a stressor (Linden et al., 1997). For instance, little detailed information regarding different time courses of physiological variables is available to date, and it has not been

* Corresponding author. Institute of Physiology, Medical University of Graz, Harrachgasse 21/5, Graz 8010, Austria. Tel.: + 433163804278 .

E-mail address: Nandu.goswami@meduni-graz.at (N. Goswami). investigated in detail whether recovery of cardiovascular and hypothalamic pituitary adrenal (HPA) activities might not only differ in duration, but also in their time courses during the first few minutes. In addition, as the regulation mechanisms during psychological stressors are fundamentally different from those during physical stressors (Berntson et al., 1994; Cacioppo et al., 1994; Lovallo, 1997; Sawchenko et al., 2000), their time-dependent behavior during recovery, too, may differ. Moreover, many people are subjected to combinations of physical and psychological stress in their working lives (e.g., Corneil et al., 1999). The combination of stressors has not been well examined, yet. While there are some studies on reactivity to combined stressors (Durocher et al., 2009; Webb et al., 2008), detailed analysis of the pattern of recovery has been rare to date.

Recovery of physical stress induced responses, particularly following exercise, has been documented (Freeman et al., 2006). The recovery of neurovascular responses following orthostatic stress, however, has been sparsely reported. Knowledge of typical time courses is relevant, for instance, to the selection of appropriate time windows for the analysis of recovery processes. Thus, there seems to 
be a relative lack of basic research on the issue of physiological recovery altogether.

Although responses to acute stress in the laboratory are not of clinical importance in themselves, they may index the way that individuals respond to ordinary psychological demands in daily life, and accumulation of inadaptive responses may eventually have pathophysiological significance (Chida and Hamer, 2008). This was shown in prospective studies; late heart rate recovery, for instance, was associated with higher carotid atherosclerosis 2 years later (Heponiemi et al., 2007). Meta-analyses showed that dispositional negative affect, a well-recognized factor for the development of coronary artery disease (Frasure-Smith and Lesperance, 2005; Kubzansky et al., 2005), is associated with poor cardiovascular recovery, independently of reactivity (Chida and Hamer, 2008). Thus, inefficient recovery may eventually result in unfavorable physical sequelae. On the other hand, the observation of bradycardia below pre-stimulus levels after the removal of mental challenge (Callister et al., 1992) might also have cardiological implications, because myocardial infarction often occurs at low heart rates (Deanfield et al., 1984). In this connection, it seems relevant to also describe typical patterns of recovery in young and healthy individuals. We believe that the rate of recovery to these orthostatic and mental challenges may have practical implications, particularly in the treatment, prognosis and outcome of patients with cardiovascular diseases and myocardial ischemia.

The mechanisms of cardiovascular regulation have been reported to be different under orthostatic and mental stress. We hypothesized that a different pattern of early term recovery responses between these stressors, when done singly or in combination, would be seen. In addition, we hypothesized that cardiovascular responses would not return to baseline for prolonged periods following the stress applications and that the effects of these stressors would continue up to at least $30 \mathrm{~min}$.

We investigated the time course of recovery of the cardiovascular (early and late) and autonomic (late) responses, induced by orthostatic and mental challenge, when applied singly or in combination. Participants were drawn from our previous study (Lackner et al., in press in which we studied reactivity responses to HUT, MA and MA+ HUT. In this companion paper, we investigated the recovery responses, particularly as there is evidence that hemodynamic responses due to stressors (in other words, physiological mechanisms activated during stress) may not be the same as recovery patterns (Gregg et al., 1999). The responses analyzed include heart rate, stroke volume, cardiac output, blood pressures and autonomic activity. In most studies, recovery was followed for 5-10 min only (Boutcher et al., 2001; Gregg et al., 1999). Individual differences in recovery at more extended periods are probably even more relevant to possible health related effects than those very shortly after the stressor (Gregg et al., 1999). To our knowledge, only one study has examined the post stress responses for longer than $10 \mathrm{~min}$ (Steptoe et al., 2003). As considerable individual differences in cardiovascular recovery can be well expected until $10 \mathrm{~min}$ after termination of the stress situation (Key et al., 2008; Linden et al., 1997), we obtained measures of cardiovascular recovery for $30 \mathrm{~min}$ after termination of the stress.

\section{Methodology}

\subsection{Participants}

We focused on healthy men whose age and physical characteristics were homogeneous because gender, age and athletic training may affect orthostatic stress responses (Goswami et al., 2008). Participants abstained from coffee and other stimulants for 2 days before the test sessions, as well as unusual exercise activities in the week prior to the study. The study was carried out in 15 healthy, non-obese, nonsmoking, non-medicated Caucasian men of moderate physical fitness, free from any somatic or mental condition $(25 \pm 3$ years, $73 \pm 7 \mathrm{~kg}$,
$180 \pm 7 \mathrm{~cm}$, supine resting heart rate $60 \pm 6 \mathrm{bpm}$; mean $\pm S D$ ). Each participant was paid 40 Euro per session.

Participants were familiarized with the test protocol and gave written informed consent to participate in the study. The study was approved by the Graz University Ethics Board and was performed in accordance with the 1989 WMA Declaration of Helsinki.

\subsection{Study design}

We used a symmetric, crossover within-subjects design and the participants were randomly allocated to each protocol. The participants served as their own control. We asked participants to abstain from coffee and other stimulants for 2 days before the test sessions.

Every day two participants did one of the three protocols (at 9-11 AM; 11 AM-1 PM). The protocols were randomized, open and separated by 2 weeks: a) head up tilt (HUT) alone, b) mental arithmetic (MA) in supine position and c) MA + HUT. Stress application was for $10 \mathrm{~min}$ in all the protocols.

The test was carried out in a semi-dark and quiet room, maintained at $24{ }^{\circ} \mathrm{C}$ and humidity at $55 \%$.

\subsection{Protocol HUT}

The orthostatic challenge was provided for 10 min by HUT. A 30 min supine rest preceded each experiment. At minute zero, the tilt table was brought to $70^{\circ}$ head-up position and after $10 \mathrm{~min}$ the table was returned to supine position. During the test participants were supported by an adjustable footrest and were instructed to avoid undue movements of the lower limbs and to breathe normally.

Since the aim of the experiment was to induce orthostatic stress without inducing syncope, the protocol was terminated if any of the following occurred (Goswami et al., 2009b): a) blood pressure fell below systolic $80 \mathrm{~mm} \mathrm{Hg}$, or that it dropped rapidly (systolic by $\geq 20 \mathrm{~mm} \mathrm{Hg} / \mathrm{min}$ and diastolic by $\geq 10 \mathrm{~mm} \mathrm{Hg} / \mathrm{min}$ ), or heart rate dropped by $\geq 15 \mathrm{bpm}$; and b) lightheadedness, dizziness, visual disturbances, nausea, stomach awareness, clammy skin, excessive sweating, or skin pallor. However, all participants went through all the protocols with no problems.

\subsection{Protocol Supine MA}

MA was provided by mental arithmetic. Participants added or subtracted continuously the numbers 6 or 7 , randomly, from a 2 or 3 digit number while lying supine. A new number was provided every $5 \mathrm{~s}$ to add or subtract from on a computer screen fixed at the eye of participants.

At the beginning of the selection process, participants were informed about the three protocols; however, participants were not notified in advance which protocol they would encounter on a given test day. During their first visit, the participants were familiarized with the laboratory, personnel and equipment. They received standardized verbal instructions about the protocol, tasks, and computer administered mental arithmetic at the beginning of the first session. Participants were told to solve the tasks as accurately and as fast as possible and that their answers were recorded. A timer applied additional pressure. Halfway through the mental arithmetic, they were asked to answer more correctly, irrespective of their correct answers. These procedures were designed to help reduce adaptation to the stress condition. No external feedback regarding performance during the mental arithmetic was provided during the study.

\subsection{MA + HUT protocol}

MA was started immediately upon assumption of the upright posture (HUT), and was ended when subject returned to supine position. 


\subsection{Self reported measures}

Emotional status was assessed on arrival at the laboratory using the State-Trait anxiety inventory (STAI) (Laux et al., 1981) and the General Depression scale (ADS) (Hautzinger and Bailer, 1993).

Performance (mistakes made) on the MA task was assessed and ratings of perception of stress (PSS), shortly before commencing the challenges and retrospectively at the end of MA, HUT and MA + HUT were done using a 5 point Likert scale (1: not stressful; 5: extremely stressful).

\subsection{Recording physiological stress responses}

The baseline data were collected for 30 min with the participants in supine position. During baseline the participants were requested to relax without falling asleep. After the stress period, physiological data were recorded for $45 \mathrm{~min}$.

Hemodynamic monitoring included blood pressure (upper arm oscillometry and finger plethysmography), heart rate (HR) (3-lead ECG), and thoracic impedance measurements using a Task Force Monitor $^{\circledR}$ (TFM, CNSystems, Graz, Austria). For the variables related to impedance cardiography, beat-to-beat values computed by the $\mathrm{TFM}^{\circledR}$ were used. Thoracic impedance $\mathrm{ZO}(\mathrm{t})$ and impedance variation $\mathrm{dZ}(\mathrm{t}) / \mathrm{dt}$ were used to calculate beat-to-beat stroke volume based on an improved Kubicek approach and cardiac output. Total peripheral resistance (TPR) was calculated as $80 \times$ mean arterial blood pressure/cardiac output (Gratze et al., 1998). TFM ${ }^{\circledR}$ ECG/impedance electrodes were positioned at the neck and thoracic regions, the latter at the midclavicular line at the xiphoid process level (Fortin et al., 2006).
A power spectrum analysis of heart rate variability assessed sympathovagal balance (Hilz and Dutsch, 2006). Low (LF: 0.04$0.15 \mathrm{~Hz}$ ) and high frequency (HF: $0.15-0.40 \mathrm{~Hz}$ ) power components of RR-intervals (RRI), were evaluated: HFrri (HF-HRV) is primarily mediated by parasympathetic modulation whereas LFrri (LF-HRV) is affected by both parasympathetic and sympathetic activities (Pagani et al., 1997; Stauss, 2003). Furthermore, we calculated the ratio of LF/ $\mathrm{HF}$, which is an indicator of sympathovagal balance.

\subsection{Statistical analysis}

Using typical cardiovascular changes during orthostatic loading from previous studies (Goswami et al., 2009a; Hinghofer-Szalkay et al., $2008)$, error probability $(\alpha)$ of 0.05 and power $(1-\beta)$ of 0.80 , we estimated the number of participants required to be 15 . Each protocol lasted for $75 \mathrm{~min}$.

To assess differences in the time course of recovery responses elicited by different stressors, we analyzed the last 2.5 min part of stress application (stress) and the first 10 min of post stress (early term), in 2.5 min epochs (Recovery ${ }_{1}$, Recovery 2 , Recovery R, and $_{3}$ Recovery 4 ). A detailed analysis of early term period was done in $30 \mathrm{~s}$ epochs (Recovery ${ }_{1}: T_{1}$ to $T_{5}$; Recovery $2: T_{6}$ to $T_{10}$; Recover $_{3}: T_{11}$ to $T_{15}$; and Recovery $4: T_{16}$ to $T_{20}$ ). To assess if there was a difference in the recovery phases in comparison to baseline, late effects (30 min post stress) were analyzed (Recoverys: 17.5 to $20 \mathrm{~min}$; and Recovery $_{12}: 27.5$ to $30 \mathrm{~min}$ ).

To evaluate the differences in early recovery after stress application induced by MA and HUT ( 2.5 min epochs), $5 \times 3$ analyses of variance (ANOVAs) were conducted, with phase (stress, Recovery ${ }_{1}$, Recovery 2 , Recovery $_{3}$, and Recovery 4 ) and protocol (HUT only, MA + HUT, Supine

Table 1

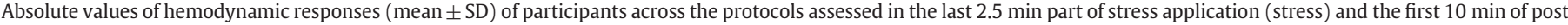

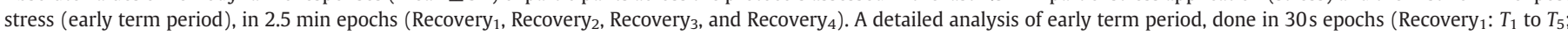

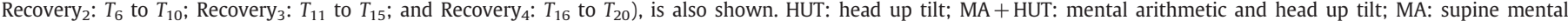
arithmetic; Recovery ${ }_{1}$ refers to first $2.5 \mathrm{~min}$, etc.

\begin{tabular}{|c|c|c|c|c|c|c|c|}
\hline & Stress & Recovery $_{1}$ & Recovery $_{2}$ & Recovery 3 & Recovery $_{4}$ & Recovery9 & Recovery $_{12}$ \\
\hline \multicolumn{8}{|c|}{ Head rate $(\mathrm{bpm})$} \\
\hline HUT & $81.0 \pm 11.2$ & $62.0 \pm 8.7$ & $56.1 \pm 7.0$ & $55.9 \pm 6.5$ & $55.5 \pm 7.2$ & $57.6 \pm 7.0$ & $58.7 \pm 7.7$ \\
\hline MA & $72.1 \pm 111$ & $68.8 \pm 8.6$ & $65.1 \pm 7.4$ & $63.3 \pm 6.9$ & $62.6 \pm 7.8$ & $63.7 \pm 7.5$ & $63.6 \pm 6.8$ \\
\hline $\mathrm{MA}+\mathrm{HUT}$ & $91.2 \pm 8.4$ & $67.2 \pm 8.0$ & $59.5 \pm 7.3$ & $59.0 \pm 8.1$ & $60.0 \pm 7.0$ & $61.3 \pm 7.2$ & $60.6 \pm 6.5$ \\
\hline \multicolumn{8}{|c|}{ Stroke volume (ml) } \\
\hline HUT & $71.3 \pm 8.3$ & $112.4 \pm 11.5$ & $116.7 \pm 10.4$ & $111.7 \pm 14.1$ & $108.9 \pm 15.3$ & $103.1 \pm 16.8$ & $101.6 \pm 16.5$ \\
\hline MA & $99.5 \pm 16.1$ & $101.1 \pm 16.6$ & $102.7 \pm 15.6$ & $102.4 \pm 14.7$ & $101.9 \pm 14.7$ & $99.0 \pm 16.9$ & $96.8 \pm 15.8$ \\
\hline $\mathrm{MA}+\mathrm{HUT}$ & $68.9 \pm 6.9$ & $111.8 \pm 11.8$ & $111.5 \pm 11.4$ & $106.4 \pm 15.3$ & $103.0 \pm 15.9$ & $98.5 \pm 17.2$ & $100.4 \pm 15.8$ \\
\hline \multicolumn{8}{|c|}{ Cardiac output (l/min) } \\
\hline HUT & $5.7 \pm 0.8$ & $6.9 \pm 1.2$ & $6.6 \pm 1.2$ & $6.2 \pm 1.1$ & $6.0 \pm 1.2$ & $5.9 \pm 1.2$ & $5.9 \pm 1.2$ \\
\hline MA & $7.1 \pm 1.5$ & $6.9 \pm 1.4$ & $6.7 \pm 1.2$ & $6.5 \pm 1.2$ & $6.4 \pm 1.2$ & $6.3 \pm 1.3$ & $6.1 \pm 1.2$ \\
\hline $\mathrm{MA}+\mathrm{HUT}$ & $6.2 \pm 0.8$ & $7.4 \pm 1.1$ & $6.6 \pm 0.9$ & $6.2 \pm 0.9$ & $6.1 \pm 0.9$ & $6.0 \pm 1.1$ & $6.1 \pm 1.0$ \\
\hline \multicolumn{8}{|c|}{ Systolic blood pressure ( $\mathrm{mmHg}$ ) } \\
\hline HUT & $118.5 \pm 11.6$ & $1221 \pm 9.8$ & $121.6 \pm 10.3$ & $121.2 \pm 1111$ & $1199 \pm 119$ & $116.1 \pm 12.1$ & $116.9 \pm 12.6$ \\
\hline MA & $128.7 \pm 12.8$ & $125.7 \pm 11.5$ & $123.7 \pm 11.4$ & $124.2 \pm 13.6$ & $123.2 \pm 13.2$ & $118.4 \pm 11.8$ & $121.3 \pm 9.3$ \\
\hline $\mathrm{MA}+\mathrm{HUT}$ & $127.9 \pm 18.8$ & $132.9 \pm 15.9$ & $127.5 \pm 12.7$ & $124.5 \pm 9.8$ & $123.1 \pm 9.2$ & $120.4 \pm 10.1$ & $121.0 \pm 11.2$ \\
\hline \multicolumn{8}{|c|}{ Mean arterial pressure ( $\mathrm{mmHg}$ ) } \\
\hline HUT & $91.5 \pm 11.9$ & $88.5 \pm 10.4$ & $86.8 \pm 9.9$ & $87.1 \pm 10.1$ & $86.6 \pm 11.4$ & $87.3 \pm 10.6$ & $87.4 \pm 10.5$ \\
\hline MA & $97.7 \pm 11.6$ & $94.4 \pm 10.8$ & $92.5 \pm 10.0$ & $92.9 \pm 11.3$ & $92.1 \pm 11.2$ & $88.1 \pm 10.9$ & $90.7 \pm 7.9$ \\
\hline $\mathrm{MA}+\mathrm{HUT}$ & $100.8 \pm 14.7$ & $98.6 \pm 10.6$ & $94.0 \pm 10.2$ & $92.5 \pm 9.2$ & $92.0 \pm 8.5$ & $91.3 \pm 10.4$ & $91.8 \pm 11.6$ \\
\hline \multicolumn{8}{|c|}{ Diastolic blood pressure ( $\mathrm{mmHg}$ ) } \\
\hline HUT & $76.9 \pm 10.3$ & $70.8 \pm 9.2$ & $69.1 \pm 8.2$ & $70.0 \pm 8.2$ & $70.1 \pm 9.6$ & $73.5 \pm 9.4$ & $73.0 \pm 9.8$ \\
\hline MA & $81.7 \pm 11.3$ & $78.9 \pm 11.0$ & $78.0 \pm 10.0$ & $78.5 \pm 10.9$ & $78.0 \pm 11.2$ & $74.8 \pm 10.6$ & $77.6 \pm 71$ \\
\hline $\mathrm{MA}+\mathrm{HUT}$ & $87.0 \pm 13.0$ & $81.3 \pm 9.1$ & $77.3 \pm 10.1$ & $76.8 \pm 9.9$ & $76.7 \pm 9.7$ & $76.8 \pm 10.0$ & $77.3 \pm 10.5$ \\
\hline \multicolumn{8}{|c|}{ Total peripheral resistance $\left(\right.$ dyn $\mathrm{s} / \mathrm{cm}^{5}$ ) } \\
\hline HUT & $1275 \pm 288$ & $1041 \pm 269$ & $1066 \pm 258$ & $1124 \pm 269$ & $1160 \pm 306$ & $1196 \pm 286$ & $1191 \pm 287$ \\
\hline MA & $1129 \pm 345$ & $1116 \pm 322$ & $1126 \pm 297$ & $1159 \pm 298$ & $1174 \pm 334$ & $1147 \pm 314$ & $1197 \pm 274$ \\
\hline $\mathrm{MA}+\mathrm{HUT}$ & $1286 \pm 282$ & $1070 \pm 227$ & $1134 \pm 219$ & $1181 \pm 203$ & $1196 \pm 213$ & $1230 \pm 289$ & $1225 \pm 299$ \\
\hline
\end{tabular}


MA) as within-subjects factors, and the cardiovascular measures as the dependent variables, followed by post hoc tests (Tukey's Honestly Significant Difference, HSD). Addition $5 \times 3$ analyses of variance (ANOVAs), with phase (recovery ${ }_{T 1-T 5}$ and recovery ${ }_{T 6-T 10}$ ) and protocol as within-subjects factors were conducted to observe recovery responses of the stressors with higher time resolution of $30 \mathrm{~s}$. The multivariate approach to repeated measures analyses was used in case of violation of the sphericity assumption, which allows valid tests under nonsphericity conditions (Vasey and Thayer, 1987).

To evaluate the differences in late recovery after stress application induced by MA and HUT, $4 \times 3$ analyses of variance (ANOVAs) was conducted, with phase (baseline, recovery ${ }_{4}$, recoverys, recovery ${ }_{12}$ ) and protocol (HUT only, MA + HUT, Supine MA) as within-subjects factors, and the cardiovascular and autonomic responses as the dependent variables, followed by post hoc tests (Tukey's Honestly Significant Difference, HSD). A natural logarithmic transformation was applied to the LF, HF and sympathovagal balance (LF/HF) data, as their distribution was skewed.

For comparing emotional stress (ADS, STAI) between the protocols, ANOVA for repeated measurements were used. The nonparametric Friedman test was used to analyze perception of stress (PSS) between the baselines of the protocols. Similarly, the difference in PSS between during- and beginning of the protocols was compared.

\section{Results}

\subsection{Early term period (Table 2)}

Means and SD of hemodynamic variables during early term period are shown in Table 1.
Results presentation (below) is sequential: beginning with main effects and followed with the interaction. Results are shown firstly in 2.5 min blocks and then detailed in 30 s epochs.

HR and DBP differed between protocols when comparing the last quarter of stress until 10 min post stress. The analysis for a higher (30 s) time resolution indicated a significant main effect of protocol on $\mathrm{HR}, \mathrm{SV}$, cardiac output and diastolic BP in the first 2.5 min of recovery. The main effect of protocol holds for HR, SV and DBP in the second quarter (from $2.30 \mathrm{~min}$ to 5:00 min of recovery).

The 2.5 min time resolution analysis showed a significant main effect of phase on HR, SV, CO, SBP, MAP, DBP and TPR. Analysis with the higher time resolution of $30 \mathrm{~s}$ revealed a significant main effect of phase on HR, SV, CO, SBP, MAP and DBP in the first 2.5 min of recovery. The main effect of phase holds for HR and CO in the second period (2.30 min to $5: 00 \mathrm{~min}$ ).

For a time resolution of $2.5 \mathrm{~min}$ the interaction phase $\times$ protocol proved to be significant for HR, SV and CO. Detailed analysis of the interaction phase $\times$ protocol with higher time resolution of $30 \mathrm{~s}$ revealed a significant effect for HR, SV and CO in first 2.5 min of recovery. No significant effect was seen in the following $2.5 \mathrm{~min}$. (Table 2).

Post hoc tests indicated no differences in the baseline of all cardiovascular variables. The post hoc tests with time resolution of 2.5 min for main effect phase indicated a significant HR decrease from stress to recovery ${ }_{1}$ and from recovery ${ }_{1}$ to recovery ${ }_{2}$ in HUT, MA + HUT and MA (Fig. 1a).

The post hoc tests with the time resolution of $2.5 \mathrm{~min}$ for the main effect phase showed, that TPR decreased significantly from stress to recovery $_{1}$ in protocol HUT and MA+HUT. Whereas in HUT no significant difference was seen between the other phases, the increase in protocol MA + HUT from recovery ${ }_{1}$ to recovery 2 was, however, significant (Fig. 1b).

Table 2

Statistical analysis of hemodynamic variables across protocols.

\begin{tabular}{|c|c|c|c|c|c|c|}
\hline & \multicolumn{2}{|c|}{$\begin{array}{l}\text { Stress, Recovery }{ }_{1}, \text { Recovery }_{2} \text {, } \\
\text { Recovery }_{3}, \text { Recovy }_{4}\end{array}$} & \multicolumn{2}{|c|}{ Recovery $_{1: T 1, T 2, T 3, T 4, T 5}$} & \multicolumn{2}{|c|}{ 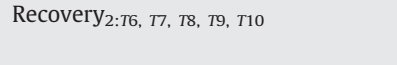 } \\
\hline \multicolumn{7}{|c|}{ Heart rate (bpm) } \\
\hline Protocol & $F(2,13)=5.2$ & $p=0.021$ & $F(2,13)=5.2$ & $p=0.022$ & $F(2,13)=8.3$ & $p=0.005$ \\
\hline Interaction & $F(8,7)=13.8$ & $p=0.001$ & $F(8,7)=7.9$ & $p<0.007$ & $F(8,7)=0.2$ & $p=0.982$ \\
\hline \multicolumn{7}{|c|}{ Stroke volume (ml) } \\
\hline Protocol & $F(2,13)=1.9$ & $p=0.182$ & $F(2,13)=5.0$ & $p<0.024$ & $F(2,13)=5.7$ & $p=0.017$ \\
\hline \multicolumn{7}{|c|}{ Cardiac output (I/min) } \\
\hline Protocol & $F(2,13)=0.6$ & $p=0.587$ & $F(2,13)=4.8$ & $p=0.027$ & $F(2,13)=0.3$ & $p=0.761$ \\
\hline Phase & $F(4,11)=19.7$ & $p<0.601$ & $F(4,11)=9.2$ & $p=0.002$ & $F(4,11)=7.2$ & $p=0.004$ \\
\hline Interaction & $F(8,7)=4.2$ & $p=0.037$ & $F(8,7)=14.6$ & $p=0.001$ & $F(8,7)=0.9$ & $p=0.558$ \\
\hline \multicolumn{7}{|c|}{ Systolic blood pressure $(\mathrm{mmHg})$} \\
\hline Protocol & $F(2,13)=3.8$ & $p=0.051$ & $F(2,13)=3.6$ & $p=0.056$ & $F(2,13)=3.6$ & $p=0.056$ \\
\hline Phase & $F(4,11)=13.8$ & $p<0.001$ & $F(4,11)=9.3$ & $p=0.002$ & $F(4,11)=0.8$ & $p=0.567$ \\
\hline Interaction & $F(8,7)=0.6$ & $p=0.760$ & $F(8,7)=1.1$ & $p=0.446$ & $F(8,7)=0.7$ & $p=0.658$ \\
\hline \multicolumn{7}{|c|}{ Diastolic blood pressure ( $\mathrm{mmHg}$ ) } \\
\hline Protocol & $F(2,13)=7.8$ & $p=0.606$ & $F(2,13)=8.2$ & $p=0.005$ & $F(2,13)=9.2$ & $p=0.003$ \\
\hline Phase & $F(4,11)=12.6$ & $p<0.001$ & $F(4,11)=11.6$ & $p=0.001$ & $F(4,11)=0.8$ & $p=0.575$ \\
\hline Interaction & $F(8,7)=22$ & $p=0.160$ & $F(8,7)=2.4$ & $p=0.129$ & $F(8,7)=0.6$ & $p=0.775$ \\
\hline \multicolumn{7}{|c|}{ Total peripheral resistance (dyn $\mathrm{s} / \mathrm{cm}^{5}$ ) } \\
\hline Protocol & $F(2,13)=0.5$ & $p=0.594$ & $F(2,13)=2.3$ & $p=0.140$ & $F(2,13)=2.4$ & $p=0.126$ \\
\hline Phase & $F(4,11)=9.5$ & $p=0.001$ & $F(4,11)=3.4$ & $p=0.050$ & $F(4,11)=1.8$ & $p=0.195$ \\
\hline Interaction & $F(8,7)=2.9$ & $p=0.090$ & $F(8,7)=1.5$ & $p=0.316$ & $F(8,7)=0.4$ & $p=0.869$ \\
\hline
\end{tabular}



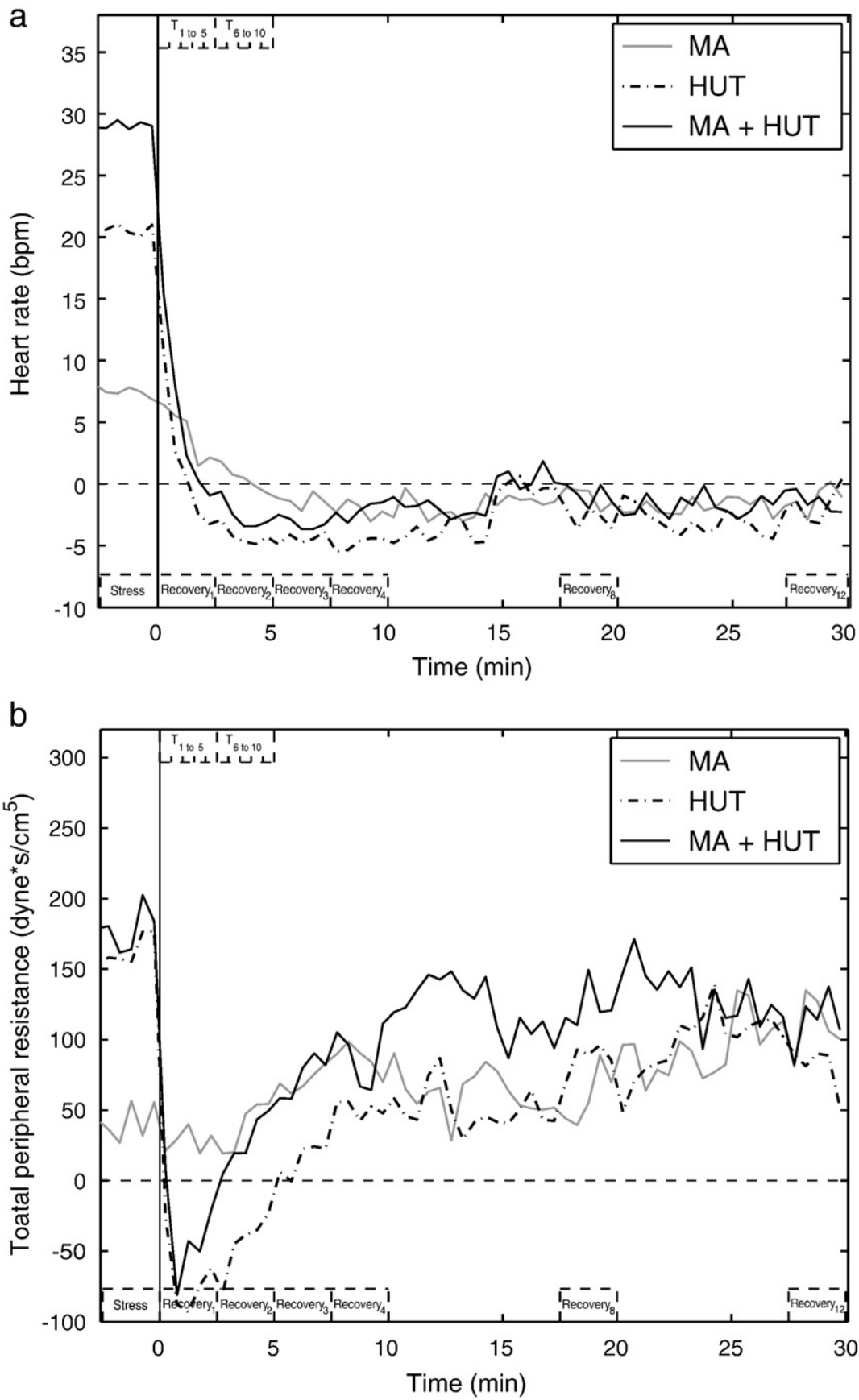

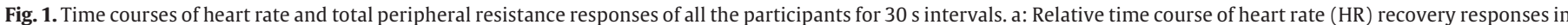

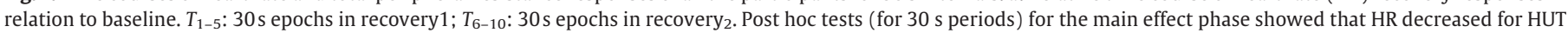

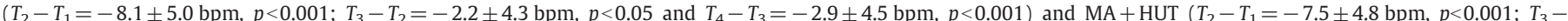

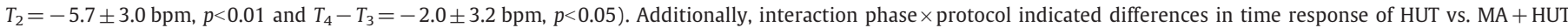

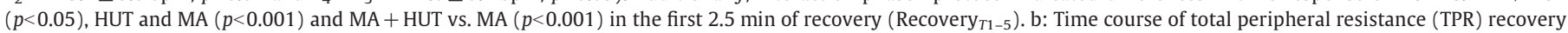
responses.

The post hoc tests with the time resolution of $2.5 \mathrm{~min}$ for the main effect phase showed, that the MAP decreased significantly from stress to recovery ${ }_{1}$ in protocol HUT and supine MA whereas in protocol MA + HUT the MAP decreased significantly from Recovery to Recovery 2 . No significant differences for the periods Recovery ${ }_{T 1-5}$ and Recovery ${ }_{T 6-10}$, were seen in the time course of MAP (interaction phase $\times$ protocol for HUT, MA + HUT and supine MA) (Fig. 2a).

Post hoc tests with time resolution of $2.5 \mathrm{~min}$ for the main effect phase showed that DBP decreased significantly from stress to recovery $T_{1}$ in all protocols (Fig. $2 \mathrm{~b}$ ).
Fig. 3 depicts HR, SV and TPR recovery responses for the period 330 s (last 30 s of stress and first 300s of recovery) across stressors. Commencements of recovery periods are shown on the right hand side of the cube; behavior of these cardiovascular recovery responses can be followed continuously (circles depict 30 s intervals). The shadowed area and hatch marks at the bottom of the figure panel represent stroke volume and heart rate, from which cardiac output can be calculated. When cardiac output is seen in relation to the other variable in the cube (total peripheral resistance) the mean arterial pressure can be obtained, according to the relationship: $\mathrm{MAP} \sim \mathrm{TPR} \times \mathrm{SV} \times \mathrm{HR}$. 


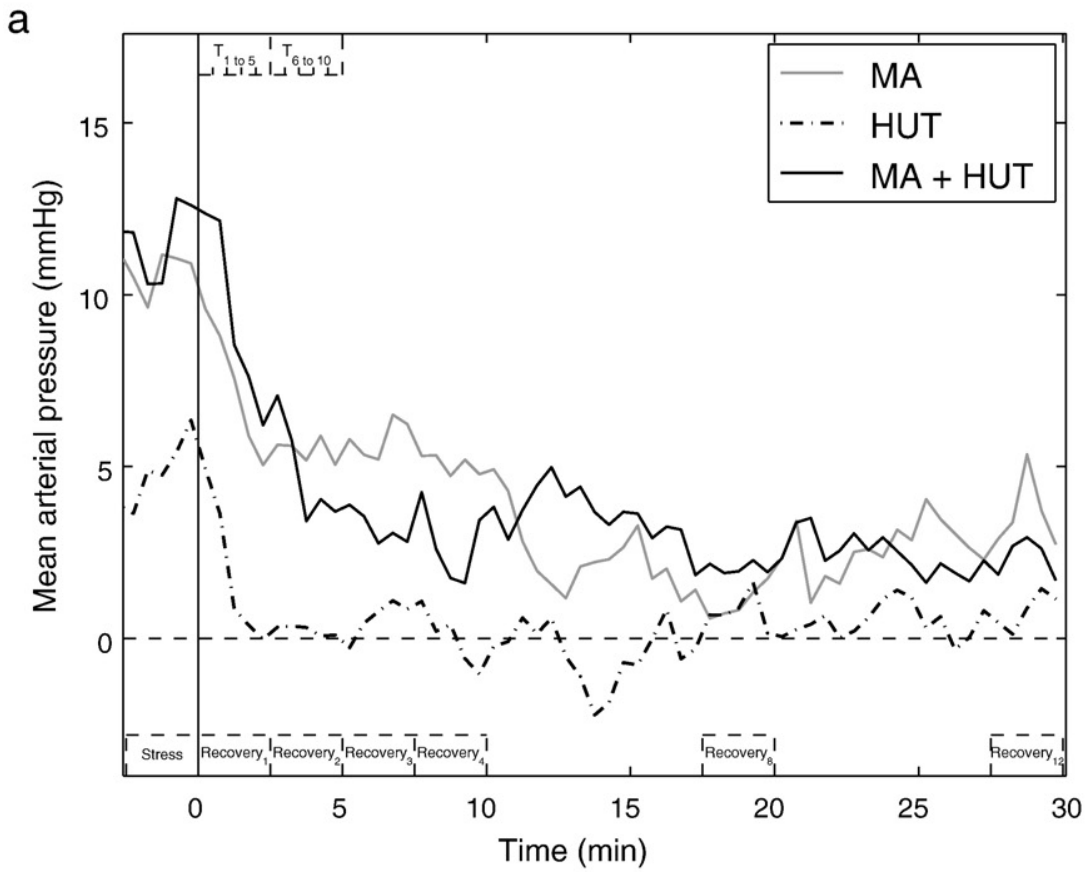

b

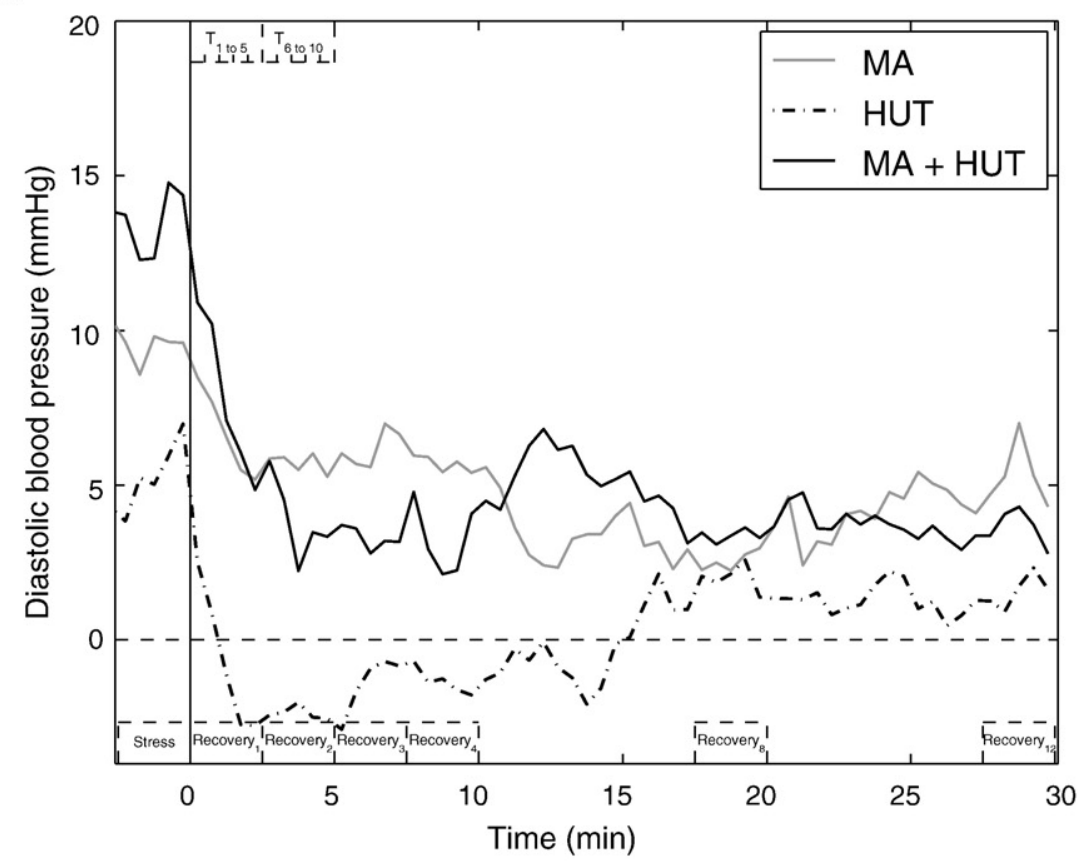

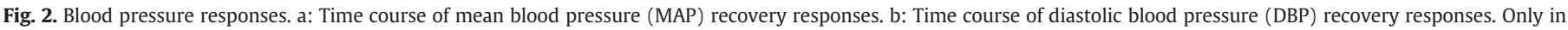
protocol MA + HUT was the decrease in DBP from Recovery ${ }_{1}$ to Recovery 2 significant.

\subsection{Late recovery (Table 3)}

Table 3 shows relative changes in hemodynamic and autonomic variables in relation to baseline (mean $\pm S D$ ) for the period late recovery.

Main effect of protocol showed significant response in HR only.

For the main effect of phases HR, SV, CO, SBP, MAP, TPR, LF component and LF/HF of HR variability showed differences between baseline and overall recovery period (Recovery $4,8,12$ ). During this period, the interaction (behavior of these responses) did not show any difference.

Overall, perception of stress (PSS) increased in response to mental challenge but postural changes did not affect stress perception. More- over, no significant differences were seen in the mistakes made between the mental arithmetic sessions or in the emotional states (ADS and STAI), which were separated by 2 weeks.

\section{Discussion}

Application of single or combined stress challenges led to stressorand time-dependent cardiovascular recovery responses. In this study, the main differences between cardiovascular recovery responses after single vs. combined stress, particularly heart rate, stroke volume and cardiac output, were seen in the first $2.5 \mathrm{~min}$ following stress termination. In the late recovery period, cardiac output was below pre-stress levels. Our findings confirm that stress effects continue for 

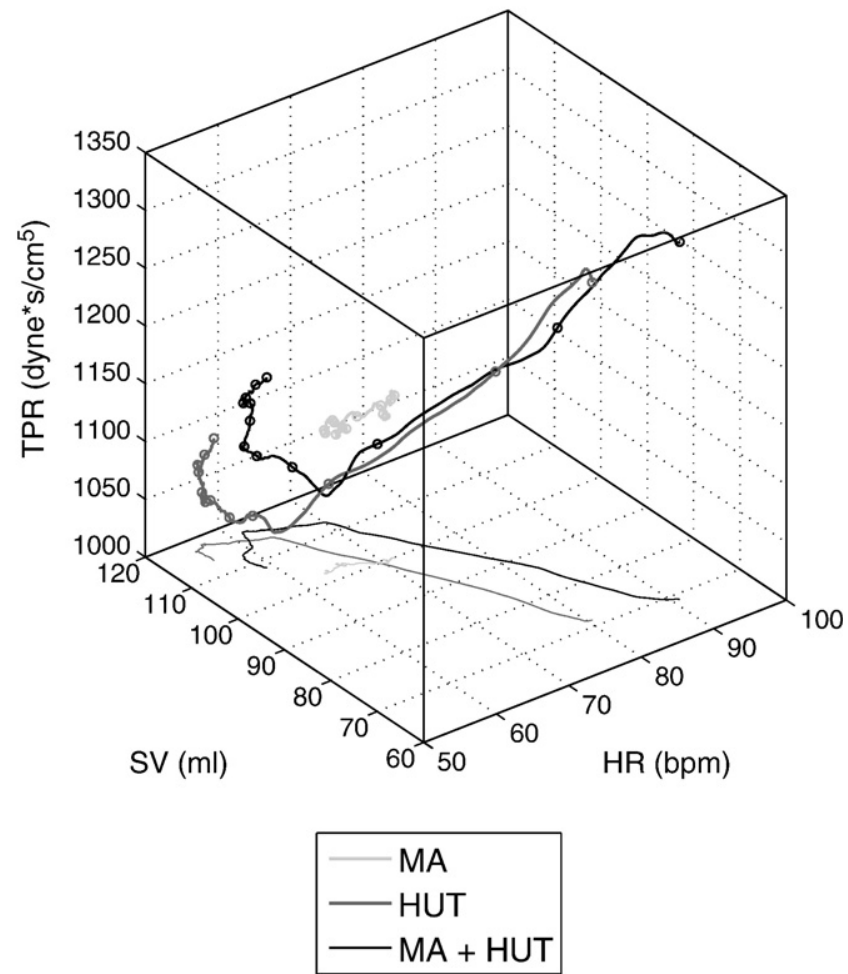

Fig. 3. Cubical representation of the moving average of heart rate (HR), stroke volume (SV) and total peripheral resistance (TPR) (behavior of these hemodynamic responses) for a period of 330 s (last 30 s of stress and first 300 s of recovery) across stressors. Commencements of recovery periods are shown on the right hand side of the cube. Circles depict 30 s intervals. The shadowed area and hatch marks at the bottom of the figure panel represent stroke volume and heart rate, from which cardiac output can be calculated. When cardiac output is seen in relation to the other variable in the cube (total peripheral resistance) the mean arterial pressure can be obtained, according to the relationship: $\mathrm{MAP} \sim \mathrm{TPR} \times \mathrm{SV} \times \mathrm{HR}$.

at least 30 min after the stress application is over and provide information on the recovery phase after mental vs. orthostatic challenge when applied singly or in combination. The underlying mechanisms in the rate of recovery may have implications for the development, recovery and outcome of cardiovascular diseases.

\subsection{Early term recovery}

The time course of heart rate was different between stressors in the first $2.5 \mathrm{~min}$ of recovery, despite having different heart rate reactivities. Exponential decreases in heart rate following termination of physical activity have been previously shown (Freeman et al., 2006). Differences in the time courses of the stroke volume responses were seen in the first 2.5 min when comparing mental challenge with orthostatic challenge or combinations of both. This is not surprising as posture changes, present in orthostatic and combined protocol, are associated with reductions in stroke volume (Goswami et al., 2009c). Upon resumption of the supine posture, and as shown in the initial recovery phase, the increases in stroke volume could be attributed to increased venous return. These differences in recovery of heart rate and stroke volume (Fig. 1a; Table 1) affected the time course of cardiac output response for periods up to $10 \mathrm{~min}$. This implies that when studying, for example, effects of mental challenge on orthostatic responses longer recovery periods are required to observe the differences in the recovery time courses of these stressors. This may probably explain the contrasting results obtained by other studies.

On the other hand, blood pressure variables (mean arterial pressure, diastolic and systolic) showed no differences in the time courses of recovery between the protocols. This confirms previous observations that arterial blood pressure is the primary regulated variable during stress applications (Julius, 1988). When compared to stress induced mean arterial pressure increases, the pressure levels were lower in the recovery phase. However, average blood pressure remained elevated above baseline values for the entire duration of the recovery, as has previously been reported (Steptoe et al., 2003). The average increases in diastolic pressure, above baseline values, during the entire recovery period have clinical significance (Fig. 1b): late diastolic blood pressure recovery responses have been associated with increased risk of hypertension (Borghi et al., 1986).

\subsection{Late recovery}

Heart rate decreases were seen at 10 min following stress termination for all the stressors. This confirms bradycardia reported in some studies following mental challenge (Callister et al., 1992) (Steptoe et al., 2003). Since myocardial infarction often occurs at low heart rates (Deanfield et al., 1984), our findings have cardiological implications.

Relative to baseline, stroke volume showed no differences at $10 \mathrm{~min}$ following the stress terminations. However, 20 and 30 min following stress termination, stroke volume was lower than the baseline. Taken together in relation to heart rate changes, the cardiac output was lower in orthostatic and combined protocols at $10 \mathrm{~min}$. As the data in Tables 2 and 3 show, mental challenge induced stroke volume responses were minimal and therefore contributed to the observed results in the cardiac output. What is rather surprising is that cardiac output was lower at 20 and 30 min post stress application. What effects might this have on, for example, renal or hepatic blood flow, especially in persons with renal or hepatic diseases? This could have important implications in prognosis of chronic renal diseases or myocardial ischemia. Complimentary cerebral blood studies are needed to observe if there is similar reduction in the cerebral blood flow.

During recovery, total peripheral resistance was higher than the baseline for all the protocols, as has been previously reported (Gregg et al., 1999)(Steptoe et al., 2003). This could be attributed to the increased sympathetic activity (LF component of HR variability) observed during this period. As the increased peripheral resistance contributes to the maintenance of blood pressure (Goswami et al., 2009c), it is not surprising that when comparing post stress effects of different stressors, mean arterial and diastolic pressures were higher after $10 \mathrm{~min}$, particularly in the combined protocol. This also has clinical application, as total peripheral resistance (increased in hypertensives) responses have been linked with markers of disease risk (Goldberg et al., 1996). Moreover, the increased peripheral resistance-which leads to increased after-load-has been associated with mental stress induced myocardial ischemia in both normal healthy volunteers and persons with coronary artery disease (see Strike and Steptoe, 2003).

The observed early as well as late responses after mental vs. orthostatic stress were different, which was expected (Kamiya et al., 2000). In particular, baroreflex function can be modulated by behavior/ mental challenge at relay sites in the medulla, pons and hypothalamus (Stephenson, 1984). Indeed, the mechanisms of cardiovascular regulation have been reported to be different in the two (orthostatic and mental) forms of stress: cardiopulmonary baroreceptor unloading due to central hypovolemia occurs with orthostatic stress while an increase in central command and arterial baroreceptor loading is noticed under mental stress (Sweene et al., 1995).

\section{Conclusions}

Recovery of hemodynamic responses, either due to single or combined stress challenges, showed stressor- and time-dependent patterns. Therefore, one cannot generalize recovery during challenges, as each stressor seems to have different time courses of recovery of the 
Table 3

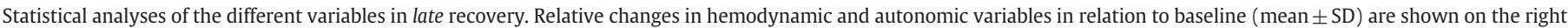

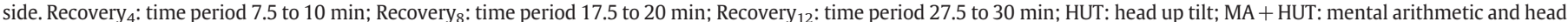
up tilt; MA: supine mental arithmetic; ln LF: natural logarithm of low freq. component; ln HF: natural logarithm of high freq. component Recovery 1 refers to first 2.5 min, etc.

\begin{tabular}{|c|c|c|c|c|c|c|}
\hline & & & & $\Delta$ Recovery $_{4}$ & $\Delta$ Recovery $_{8}$ & $\Delta$ Recovery $_{12}$ \\
\hline \multicolumn{7}{|c|}{ Heart rate ( $\mathrm{bpm})$} \\
\hline Protocol & $F(2,13)=7.2$ & $p=0.008$ & HUT & $-4.9 \pm 3.2$ & $-2.8 \pm 4.0$ & $-1.7 \pm 4.0$ \\
\hline Phase & $F(3,12)=6.2$ & $p=0.009$ & MA & $-2.1 \pm 4.6$ & $-1.0 \pm 4.3$ & $-1.1 \pm 4.8$ \\
\hline Interaction & $F(6,9)=1.0$ & $p=0.465$ & $\mathrm{MA}+\mathrm{HUT}$ & $-2.2 \pm 4.8$ & $-0.9 \pm 4.9$ & $-1.5 \pm 4.9$ \\
\hline \multicolumn{7}{|c|}{ Stroke volume $(\mathrm{ml})$} \\
\hline Protocol & $F(2,13)=1.6$ & $p=0.238$ & HUT & $3.6 \pm 7.6$ & $-2.2 \pm 4.4$ & $-3.6 \pm 6.3$ \\
\hline Phase & $F(3,12)=14.2$ & $p<0.001$ & MA & $0.3 \pm 7.7$ & $-2.6 \pm 8.4$ & $-4.8 \pm 7.6$ \\
\hline Interaction & $F(6,9)=1.4$ & $p=0.315$ & $\mathrm{MA}+\mathrm{HUT}$ & $-1.3 \pm 8.4$ & $-5.8 \pm 6.6$ & $-3.9 \pm 5.5$ \\
\hline \multicolumn{7}{|c|}{ Cardiac output (I/min) } \\
\hline Protocol & $F(2,13)=1.1$ & $p=0.362$ & HUT & $-0.3 \pm 0.6$ & $-0.4 \pm 0.5$ & $-0.4 \pm 0.6$ \\
\hline Phase & $F(3,12)=4.9$ & $p=0.019$ & MA & $-0.2 \pm 0.6$ & $-0.3 \pm 0.6$ & $-0.4 \pm 0.7$ \\
\hline Interaction & $F(6,9)=0.5$ & $p=0.767$ & $\mathrm{MA}+\mathrm{HUT}$ & $-0.4 \pm 0.6$ & $-0.5 \pm 0.5$ & $-0.4 \pm 0.6$ \\
\hline \multicolumn{7}{|c|}{ Systolic blood pressure ( $\mathrm{mmHg}$ ) } \\
\hline Protocol & $F(2,13)=1.2$ & $p=0.339$ & HUT & $1.2 \pm 10.2$ & $-2.6 \pm 7.9$ & $-1.8 \pm 8.9$ \\
\hline Phase & $F(3,12)=6.7$ & $p=0.007$ & MA & $5.0 \pm 6.8$ & $0.1 \pm 9.3$ & $3.0 \pm 8.2$ \\
\hline Interaction & $F(6,9)=1.0$ & $p=0.464$ & $\mathrm{MA}+\mathrm{HUT}$ & $0.5 \pm 11.0$ & $-2.2 \pm 7.8$ & $-1.6 \pm 7.4$ \\
\hline \multicolumn{7}{|c|}{ Mean arterial pressure ( $\mathrm{mmHg}$ ) } \\
\hline Protocol & $F(2,13)=1.5$ & $p=0.262$ & HUT & $0.0 \pm 9.2$ & $0.8 \pm 6.5$ & $0.8 \pm 7.2$ \\
\hline Phase & $F(3,12)=4.5$ & $p=0.024$ & MA & $5.1 \pm 6.4$ & $1.0 \pm 8.1$ & $3.6 \pm 7.1$ \\
\hline Interaction & $F(6,9)=1.0$ & $p=0.469$ & $\mathrm{MA}+\mathrm{HUT}$ & $2.7 \pm 8.5$ & $2.0 \pm 7.4$ & $2.5 \pm 7.3$ \\
\hline \multicolumn{7}{|c|}{ Diastolic blood pressure ( $\mathrm{mmHg}$ ) } \\
\hline Protocol & $F(2,13)=1.9$ & $p=0.185$ & HUT & $-1.3 \pm 9.3$ & $2.0 \pm 6.0$ & $1.6 \pm 7.0$ \\
\hline Phase & $F(3,12)=3.4$ & $p=0.053$ & MA & $5.7 \pm 7.0$ & $2.5 \pm 9.4$ & $5.3 \pm 8.2$ \\
\hline Interaction & $F(6,9)=3.1$ & $p=0.062$ & $\mathrm{MA}+\mathrm{HUT}$ & $3.2 \pm 8.4$ & $3.4 \pm 8.0$ & $3.9 \pm 7.6$ \\
\hline \multicolumn{7}{|c|}{ Total peripheral resistance (dyn $\mathrm{s} / \mathrm{cm}^{5}$ ) } \\
\hline Protocol & $F(2,13)=0.3$ & $p=0.765$ & HUT & $51 \pm 162$ & $87 \pm 122$ & $80 \pm 123$ \\
\hline Phase & $F(3,12)=4.0$ & $p=0.034$ & MA & $87 \pm 167$ & $59 \pm 153$ & $110 \pm 147$ \\
\hline Interaction & $F(6,9)=0.6$ & $p=0.701$ & $\mathrm{MA}+\mathrm{HUT}$ & $89 \pm 172$ & $123 \pm 162$ & $118 \pm 168$ \\
\hline \multicolumn{7}{|l|}{ In $L F\left(m s^{2}\right)$} \\
\hline Protocol & $F(2,13)=1.0$ & $p=0.387$ & HUT & $0.10 \pm 0.64$ & $0.32 \pm 0.60$ & $0.52 \pm 0.66$ \\
\hline Phase & $F(3,12)=8.3$ & $p=0.003$ & MA & $-0.07 \pm 0.55$ & $0.37 \pm 0.46$ & $0.24 \pm 0.60$ \\
\hline Interaction & $F(6,9)=1.6$ & $p=0.244$ & $\mathrm{MA}+\mathrm{HUT}$ & $0.19 \pm 0.76$ & $0.29 \pm 1.13$ & $0.18 \pm 0.76$ \\
\hline \multicolumn{7}{|l|}{ In $H F\left(m s^{2}\right)$} \\
\hline Protocol & $F(2,13)=4.0$ & $p=0.046$ & HUT & $0.52 \pm 0.68$ & $0.42 \pm 0.72$ & $0.64 \pm 0.75$ \\
\hline Phase & $F(312)=1.1$ & $p=0.382$ & MA & $-0.24 \pm 0.72$ & $0.03 \pm 0.56$ & $-0.01 \pm 0.62$ \\
\hline Interaction & $F(6,9)=3.1$ & $p=0.065$ & $\mathrm{MA}+\mathrm{HUT}$ & $0.31 \pm 1.05$ & $0.11 \pm 1.18$ & $0.11 \pm 1.06$ \\
\hline \multicolumn{7}{|c|}{ In $(L F / H F)(-)$} \\
\hline Protocol & $F(213)=4.4$ & $p=0.035$ & HUT & $-0.46 \pm 0.58$ & $-0.12 \pm 0.51$ & $-0.17 \pm 0.35$ \\
\hline Phase & $F(312)=9.0$ & $p=0.002$ & MA & $0.12 \pm 0.66$ & $0.28 \pm 0.60$ & $0.21 \pm 0.68$ \\
\hline Interaction & $F(6,9)=1.2$ & $p=0.375$ & $\mathrm{MA}+\mathrm{HUT}$ & $-0.12 \pm 0.63$ & $0.18 \pm 0.39$ & $0.05 \pm 0.57$ \\
\hline
\end{tabular}

cardiovascular responses. This may be attributed to the stressors activating different regulatory mechanisms. In addition, our study is in agreement with the concept that effects of the physical and mental stressors induced hemodynamic responses (e.g. blood pressure and total peripheral resistance increases) could add over the entire day ('carryover') and predispose susceptible persons to hypertension (Pieper and Brosschot, 2005).

Stress specific responses are, however, not only limited to the recovery phase. For example, when comparing the reactivity of mental tasks and static tasks differences are observed, partially attributed to different adaptation processes (Seibt et al., 2001). We have also observed that there are differences in initial responses to mental, orthostatic and complex stressors (Lackner et al., in press).

\section{Acknowledgements}

We thank the volunteers for their time, patience, and collaboration in participating in this study. This study was supported by the Austrian Research Promotion Agency (FFG Project 817086 'Orthocap’).

\section{References}

Berntson, G.G., Cacioppo, J.T., Binkley, P.F., Uchino, B.N., Quigley, K.S., Fieldstone, A., 1994 Autonomic cardiac control. 3. Psychological stress and cardiac response in autonomic space as revealed by pharmacological blockades. Psychophysiology 31, 599-608.

Borghi, C., Costa, F.V., Boschi, S., Mussi, A., Ambrosioni, E., 1986. Predictors of stable hypertension in young borderline subjects -5 year follow up study. J. Cardiovasc. Pharmacol. 8, S138-S141.

Boutcher, S.H., Nurhayati, Y., McLaren, P.F., 2001. Cardiovascular response of trained and untrained old men to mental challenge. Med. Sci. Sports Exerc. 33, 659-664.

Cacioppo, J.T., Uchino, B.N., Berntson, G.G., 1994. Individual-differences in the autonomic origins of heart-rate reactivity-the psychometrics of respiratory sinus arrhythmia and preejection period. Psychophysiology 31, 412-419.

Callister, R., Suwarno, N.O., Seals, D.R., 1992. Sympathetic activity is influenced by taskdifficulty and stress perception during mental challenge in humans. J Physiol. (Lond.) 454, 373-387.

Chida, Y., Hamer, M., 2008. Chronic psychosocial factors and acute physiological responses to laboratory-induced stress in healthy populations: a quantitative review of 30 years of investigations. Psychol. Bull. 134, 829-885.

Corneil, W., Beaton, R., Murphy, S., Johnson, C., Pike, K., 1999. Exposure to traumatic incidents and prevalence of posttraumatic stress symptomatology in urban firefighters in two countries. J. Occup. Health Psychol. 4, 131-141.

Deanfield, J.E., Shea, M., Kensett, M., Horlock, P., Wilson, R.A., de Landsheere, C.M., Selwyn, A.P., 1984. Silent myocardial ischaemia due to mental stress. Lancet 3, 1001-1005. 
Durocher, J.J., Schwartz, C.E., Carter, J.R., 2009. Sympathetic neural responses to mental stress during acute simulated microgravity. J. Appl. Physiol. 107, 518-522.

Fortin, J., Habenbacher, W., Heller, A., Hacker, A., Gruellenberger, R., Innerhofer, J., Passath, H., Wagner, C., Haitchi, G., Flotzinger, D., Pacher, R., Wach, P., 2006. Non-invasive beatto-beat cardiac output monitoring by an improved method of transthoracic bioimpedance measurement. Comput. Biol. Med. 36, 1185-1203.

Frasure-Smith, N., Lesperance, F., 2005. Reflections on depression as a cardiac risk factor. Psychosom. Med. 67, S19-S25.

Freeman, J.V., Dewey, F.E., Hadley, D.M., Myers, J., Froelicher, V.F., 2006. Autonomic nervous system interaction with the cardiovascular system during exercise. Prog. Cardiovasc. Dis. 48, 342-362.

Goldberg, A.D., Becker, L.C., Bonsall, R., Cohen, J.D., Ketterer, M.W., Kaufman, P.G. Krantz, D.S., Light, K.C., McMahon, R.P., Noreuil, T., Pepine, C.J., Raczynski, J., Stone, P.H., Strother, D., Taylor, H., Sheps, D.S., 1996. Ischemic, hemodynamic, and neurohormonal responses to mental and exercise stress-experience from the psychophysiological investigations of Myocardial Ischemia Study (PIMI). Circulation 94, 2402-2409.

Goswami, N., Loeppky, J.A., Hinghofer-Szalkay, H., 2008. LBNP: past protocols and technical considerations for experimental design. Aviat. Space Environ. Med. 79 459-471.

Goswami, N., Grasser, E., Roessler, A., Schneditz, D., Hinghofer-Szalkay, H., 2009a. The cardiovascular response to lower body negative pressure in humans depends on seal location. Physiol. Res. 58, 311-318.

Goswami, N., Lackner, H.K., Grasser, E.K., Hinghofer-Szalaky, H.G., 2009b. Individual stability of orthostatic tolerance response. Acta Physiol. Hung. 96, 157-166.

Goswami, N., Roessler, A., Lackner, H.K., Schneditz, D., Grasser, E.K., Hinghofer-Szalaky, H., 2009c. Heart rate and stroke volume response patterns to augmented orthostatic stress. Clin. Auton. Res. 19, 157-165.

Gratze, G., Fortin, J., Holler, A., Grasenick, K., Pfurtscheller, G., Wach, P., et al., 1998. A software package for non-invasive, real-time beat-to-beat monitoring of stroke volume, blood pressure, total peripheral resistance and for assessment of autonomic function. Comput. Biol. Med. 28, 121-142.

Gregg, M.E., James, J.E., Matyas, T.A., Thorsteinsson, E.B., 1999. Hemodynamic profile of stress-induced anticipation and recovery. Int. J. Psychophysiol. 34, 147-162.

Hautzinger, M., Bailer, M., 1993. Allgemeine Depressions Skala. Weinheim, Beltz.

Heponiemi, T., Elovainio, M., Pulkki, L., Putonnen, S., Raitakari, O., Keltikangas-Jarvinen, L., 2007. Cardiac autonomic reactivity and recovery in predicting carotid atherosclerosis: the cardiovascular risk in young Finns study. Health Psychol. 26 $13-21$

Hilz, M.J., Dutsch, M., 2006. Quantitative studies of autonomic function. Muscle Nerve 33, 6-20.

Hinghofer-Szalkay, H., Goswami, N., Roessler, A., Grasser, E., Schneditz, D., 2008. Reactive hyperemia in the human liver. Am. J. Physiol.: Gasterointest. Liver Physiol. 295, G332-G337.

Julius, S., 1988. The blood-pressure seeking properties of the central nervous-system. J. Hypertens. 6, 177-185.

Kamiya, A., Iwase, S., Michikami, D., Fu, O., Mano, T., 2000. Head-down bed rest alters sympathetic and cardiovascular responses to mental stress. Am. J. Physiol., Regul. Integr. Comp. Physiol 279, R440-R447.
Key, B.L., Campbell, L., T., S., Bacon, S.L., Gerin, W., 2008. The influence of trait and state rumination on cardiovascular recovery from a negativeemotional stressor. J. Behav. Med. 31, 237-248.

Kubzansky, L.D., Davidson, K.W. Rozanski, A, 2005. The clinical impact ofnegative psychological states: expanding the spectrum of risk for coronary artery disease. Psychosom. Med. 67, S10-S14.

Lackner, H.K., Goswami N., Papousek, I., Roessler, A., Grasser, E.K., Montani, J.P., Jezova, D., Hinghofer-Szalkay, $\mathrm{H}$., in press. Time course of cardiovascular responses induced by mental and orthostatic challenges. Int J Psychophysiol. doi:10.1016/j. ijpsycho.2009.11.003.

Laux, L., Glanzmann, P., Schaffner, P., Spielberger, C.D., 1981. Das State-TraitAngstinventar. Weinheim, Beltz.

Linden, W., Earle, T.L., Gerin, W., Christenfeld, N., 1997. Physiological stress reactivity and recovery: conceptual siblings separated at birth? J. Psychosom. Res. 42, 117-135.

Lovallo, W.R., 1997. Stress and Health: Biological and Psychological Interactions. Sage Publications, Thousand Oaks.

Lovallo, W.R., Gerin, W., 2003. Psychophysiological reactivity: mechanisms and pathways to cardiovascular disease. Psychosom. Med. 65, 36-45.

Pagani, M., Montano, N., Porta, A., Malliani, A., Abboud, F.M., Birkett, C., Somers, V.K., 1997. Relationship between spectral components of cardiovascular variabilities and direct measures of muscle sympathetic nerve activity in humans. Circulation 95, 1441-1448.

Papousek, I., Nauschnegg, K., Paechter, M., Lackner, H.K., Goswami, N., Schulter, G., in press. Trait and state positive affect and cardiovascular recovery from experimental academic stress. Biol Psychol. doi:10.1016/j.biopsycho.2009.11.008.

Pieper, S., Brosschot, J.F., 2005. Prolonged stress-related cardiovascular activation: is there any? Ann. Behav. Med. 30, 91-103.

Sawchenko, P.E., Li, H.Y., Ericsson, A., 2000. Circuits and mechanisms governing hypothalamic responses to stress: a tale of two paradigms. Biol. Basis Mind Body Interact. 122, 61-78.

Seibt, R., Scheuch, K., Hinz, A., 2001. Short communication: reproducibility of cardiovascular responses to mental and static tasks. Stress Health 17, 245-252.

Stauss, H.M., 2003. Heart rate variability. Am. J. Physiol., Regul. Integr. Comp. Physiol. 285, R927-R931.

Stephenson, R.B., 1984. Modification of reflex regulation of blood pressure by behavior Annu. Rev. Physiol. 46, 133-142.

Steptoe, A., Willemsen, G., Kunz-Ebrecht, S.R., Owen, N., 2003. Socioeconomic status and hemodynamic recovery from mental stress. Psychophysiology 40, 184-191.

Strike, P.C., Steptoe, A., 2003. Systematic review of mental stress-induced myocardial ischaemia. Eur. Heart J. 24, 690-703.

Sweene, C.A., Bootsma, M., Van Bolhuis, H.H., 1995. Different autonomic responses to orthostatic and to mental stress in young normals. Homeostasis 36, 287-292.

Vasey, M.W., Thayer, J.F., 1987. The continuing problem of false positives in repeated measures ANOVA in psychophysiology: a multivariate solution. Psychophysiology $24,479-486$.

Webb, H.E., Weldy, M.L., Fabianke-Kadue, E.C., Orndorff, G.R., Kamimoril, G.H., Acevedo, E.O., 2008. Psychological stress during exercise: cardiorespiratory and hormonal responses. Eur. J. Appl. Physiol. 104, 973-981. 